\title{
Gaze failure, drifting eye movements, and centripetal nystagmus in cerebellar disease
}

\author{
J. LEECH, ${ }^{1}$ M. GRESTY ${ }^{1} \mathrm{~K}$. HESS, ${ }^{2}$ AND PETER RUDGE ${ }^{1}$ \\ From the ${ }^{1}$ Medical Research Council's Hearing and Balance Unit, Institute of Neurology, National Hospital, \\ Queen Square, London, WC1, and ${ }^{2}$ Neurological Clinic of the University of Zurich, Switzerland
}

SUMMARY Three abnormalities of eye movement in man are described which are indicative of cerebellar system disorder, namely, centripetally beating nystagmus, failure to maintain lateral gaze either in darkness or with eye closure, and slow drifting movements of the eyes in the absence of fixation. Similar eye movement signs follow cerebellectomy in the primate and the cat. These abnormalities of eye movement, together with other signs of cerebellar disease, such as rebound, alternating, and gaze paretic nystagmus, are explained by the hypothesis that the cerebellum helps to maintain lateral gaze and that brain stem mechanisms which monitor gaze position generate compensatory biases in the absence of normal cerebellar function.

Cerebellar disease in man causes a variety of abnormalities of eye movement, including dysmetria (Orzechowski, 1927), gaze paretic nystagmus (Holmes, 1917), and rebound nystagmus (Hood et al., 1973). Examination of the direct-coupled electro-oculographic (EOG) records obtained in the Medical Research Council's Hearing and Balance Unit has revealed three additional abnormalities not previously reported in man which we consider are also indicative of cerebellar system disorder, namely, centripetal nystagmus, in which the fast phase of the nystagmus beats towards the position of primary gaze, failure of gaze maintenance after eye closure or in darkness, and drifting movements of the eyes in the absence of fixation.

\section{Methods}

\section{PATIENTS}

All patients with centripetal nystagmus were surveyed. It was found that centripetal nystagmus occurred in patients with peripheral vestibular lesions, those intoxicated with drugs or suffering from congenital abnormalities, and in patients with disorders of the central nervous system. The characteristics of nystagmus in peripheral lesions are well documented and are discussed later. Drugintoxicated and congenitally abnormal patients were excluded from the study because of the inability to specify the lesions in these cases. The remaining 17 patients, ranging in age from 23 to 73 years, had

Address for reprints: Dr P. Rudge, Nationa! Hospital, Queen Square, London WCIN 3BG subacute or chronic disorders of the central nervous system. The only clinical feature common to all was evidence of a cerebellar system disorder, although the majority did have a wide variety of signs indicative of involvement of other systems-for example, pyramidal system, cranial nerve palsies. A detailed clinical description of the cases used to illustrate abnormalities of eye movement in this paper is given in the Appendix. Of the 17 cases 5 had a cerebellar tumour, 4 multiple sclerosis, 7 degenerative and vascular disease, and 1 Arnold-Chiari malformation.

The diagnosis of cerebellar tumour was verified at operation in all five cases; one patient had a haemangioblastoma and the remainder had astrocytomas. All the patients with a diagnosis of multiple sclerosis were classified as definite by McAlpine's criteria (McAlpine et al., 1972). Three of these had a history of relapse and remission, while the fourth had the progressive form of the disease. A diagnosis of vascular disease was made in patients who had a history with an acute onset and who had hypertension or other signs of vascular disease. Degenerative disease of the central nervous system was diagnosed in patients with a known family history, progressive neurological disturbance in patients older than 50 years, or if there was radiological evidence of atrophy of the cerebellum. The diagnosis in the patient with Arnold-Chiari malformation was confirmed at operation.

\section{Results}

CENTRIPETAL NYSTAGMUS

Centripetally beating nystagmus was present, by 

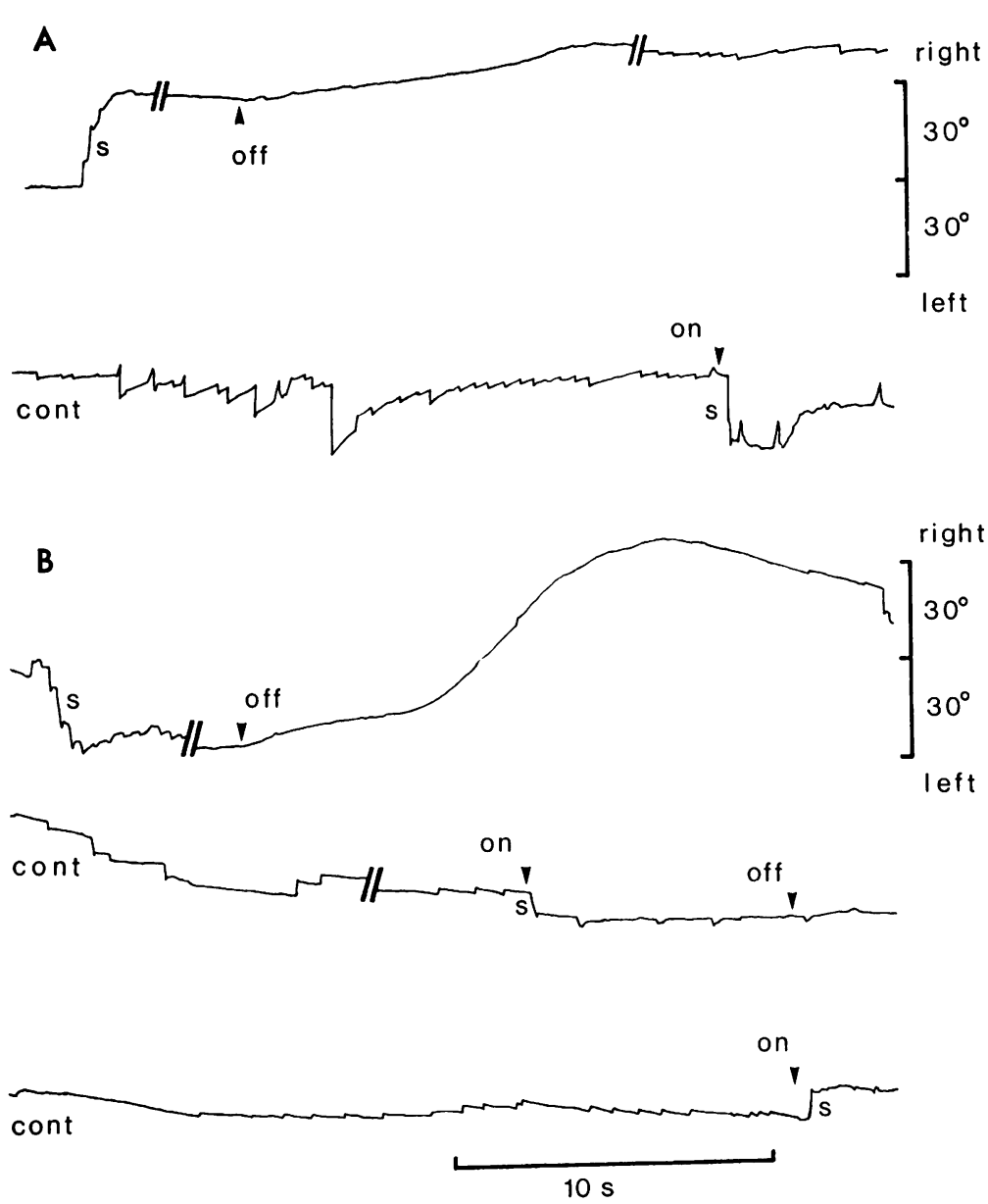

Fig. 1 EOG recordings $o$, horizontal eye movements in a patient with vascular disease (case 1).

A. The patient's eyes are initially in primary gaze, fixated on a central target light in an otherwise dark environment. A second target light appears $30^{\circ}$ to the right and the central light is extinguished. The patient executes saccadic eye movement( $s$ ), $\mathrm{s}$, to fixate the new target. Subsequently the target is turned off for 1 minute, and in total darkness the eyes drift further to the right; small saccadic movements beating to the left begin and attempts are made to correct the drift (cont $=$ continuation of the recording). Eventually a distinct nystagmus is evident. The target finally reappears $30^{\circ}$ to the right and corrective movements, $\mathrm{s}$, are made for refixation.

B. The experimental regime is the same as in $\mathrm{A}$, but this time the lateral target appears at $30^{\circ}$ left. When it is extinguished, the eyes drift to the extreme right and then back to the left with the assistance of small saccades (earliest portion of the first continuation trace). The eyes continue to drift to the left, and right-beating nystagmus develops. The target light is turned on for 4 seconds to correct the position of the eyes, $\mathrm{s}$, and a fine left-beating nystagmus is immediately evident. The target is turned off for a further 28 seconds, and in this period of darkness the second continuation trace shows that the eyes drift further to the left and a right-beating nystagmus appears. A final rightward correction, $\mathrm{s}$, is made when the target light is turned on again.

Net EOG drift estimated with reference to eye position during target refixations-1 $1 \%$ minute.

definition, in all patients in the survey. Such nystagmus occurred in the presence of optic fixation, in darkness, or on eye closure. An example of a bilateral centripetal nystagmus occurring in darkness is presented in Fig. 1. When illumination is turned off, the patient's eyes drift about in the darkness and a centripetally beating nystagmus develops. The centripetal nystagmus is immediately abolished when the illumination is turned on again and is replaced by centrifugal nystagmus (Fig. 1B). In some other patients a centripetal nystagmus was seen immediately the eyes attained an eccentric gaze position.

An example of predominantly unilateral centripetal nystagmus following eye closure is presented in
Fig. 2B. After closure there is a failure to maintain lateral gaze, and the eyes assume a more neutral position. During the movement towards the neutral position a centripetal nystagmus appears. Fifteen patients had a unilateral centripetal nystagmus, which could occur under conditions of darkness, closure, or fixation, or any combination of these. Of the 2 remaining patients one had bilateral centripetal nystagmus in darkness; the other had a unilateral centripetal nystagmus in one direction in darkness and in the other direction on eye closure.

FAILURE OF GAZE MAINTENANCE

A failure of gaze maintenance means that the eyes move rapidly from an eccentric gaze position towards 


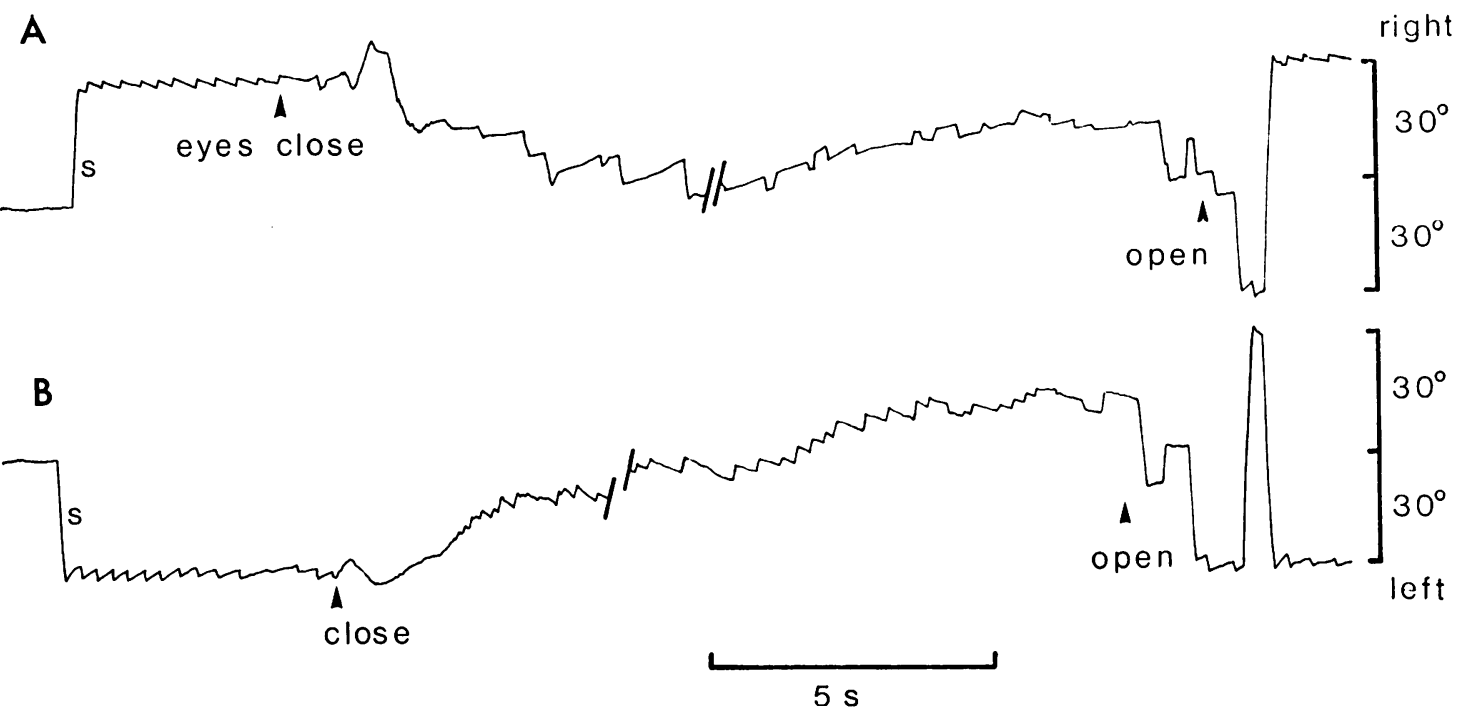

Fig. 2 EOG recordings of horizontal eye movements in a patient (case 2) after surgical removal of a tumour arising from the lateral part of the floor of the fourth ventricle and affecting the left cerebellar hemisphere.

A. Initially the eyes fixate a target light in primary gaze. A second target light appears $30^{\circ}$ to the right and the patient fixates it, $\mathrm{s}$. Immediately a right-beating nystagmus $\left(1^{\circ}\right)$ develops. After eye closure there is a rapid drift towards primary gaze, on which is superimposed a left-beating nystagmus such that the net eye movement is towards primary gaze, but the slow-phase movement is to the right. Eventually the nystagmus ceases and the eyes drift to the right and show small irregular saccadic movements with occasional isolated, nystagmic beats. The eyes open, and after a short series of inappropriate movements the $30^{\circ}$ right target is refixated and the right-beating $\left(1^{\circ}\right)$ nystagmus reappears.

B. A target $30^{\circ}$ left is fixated, $\mathrm{s}$, with the appearance of a left-beating $\left(1^{\circ}\right)$ nystagmus. After eye closure the eyes drift towards and through primary gaze. Superimposed on the drift is a right-beating nystagmus, of which the slow phase is to the left. Eventually the eyes are positioned in the right side of the orbit. When the eyes are opened, inappropriate movements are made until the eyes refixate the target and a left beating nystagmus is again seen.

EOG drift negligible.

primary gaze. The movement is 'triggered' either by closing the eyes or darkness and takes the approximate shape of an exponential decay rather than a saccade. The whole movement lasts between 2 and 6 seconds. Eleven of the patients were unable to maintain eccentric gaze on eye closure (Figs. 2 and 3), though all maintained eccentric gaze with fixation. Gaze failure was present in darkness in 2 patients, both of whom had multiple sclerosis. With eye closure the patients failed to maintain gaze to both sides except in one case with a left-sided cerebellar tumour and brain stem involvement, in which the failure was only to the left. When the eyes returned towards centre the new neutral or null position of the eyes was not necessarily the primary position. This is illustrated in Fig. 3A, where the eyes tended to come to rest some $10^{\circ}$ to the right of the primary position whether the initial gaze was to the left or right. The movement of the eyes follows an approximately exponential time course with a time constant of 0.5 to 1.5 seconds. Encouragement to maintain an eccentric position of gaze during eye closure resulted in feeble and unsuccessful corrective movements.

\section{DRIFT MOVEMENT}

The term 'drift movements' means wandering movements of the eyes, attaining velocities of only 1 or $2^{\circ}$ per second and enduring for many tens of seconds. Removal of fixation by eye closure, darkness, or both resulted in slow drifting movements of the eyes in 9 of the patients. An example occurring in darkness is shown in Fig. 1B, where the eyes move nearly $40^{\circ}$ to the right of the primary position, then drift slowly back past the midline. In other cases the drift appeared to be unidirectional, as in Fig. 2A and B, where there is a slow drift to the right of the midline. In the 5 cases in which there was evidence of a predominantly unilateral lesion the drift tended to be away from that side. Furthermore, in those cases where there was a failure to maintain eccentric gaze and the neutral position was displaced to one side of the position of primary gaze, the drift was also predominantly to the same side of the midline as this 

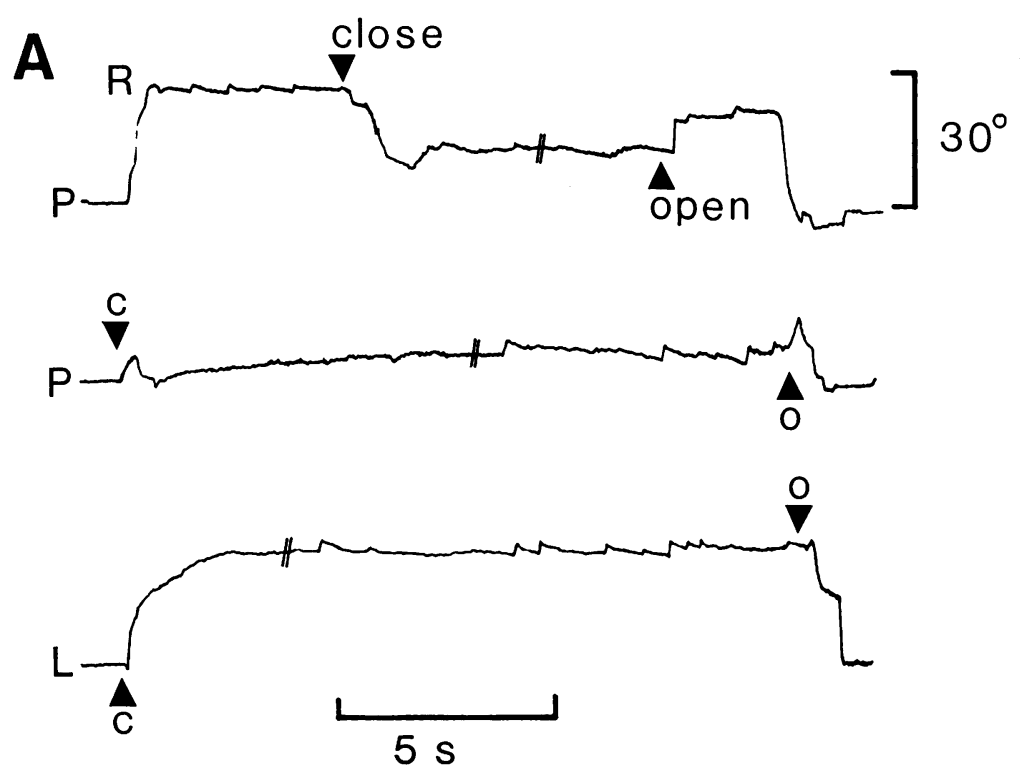

B
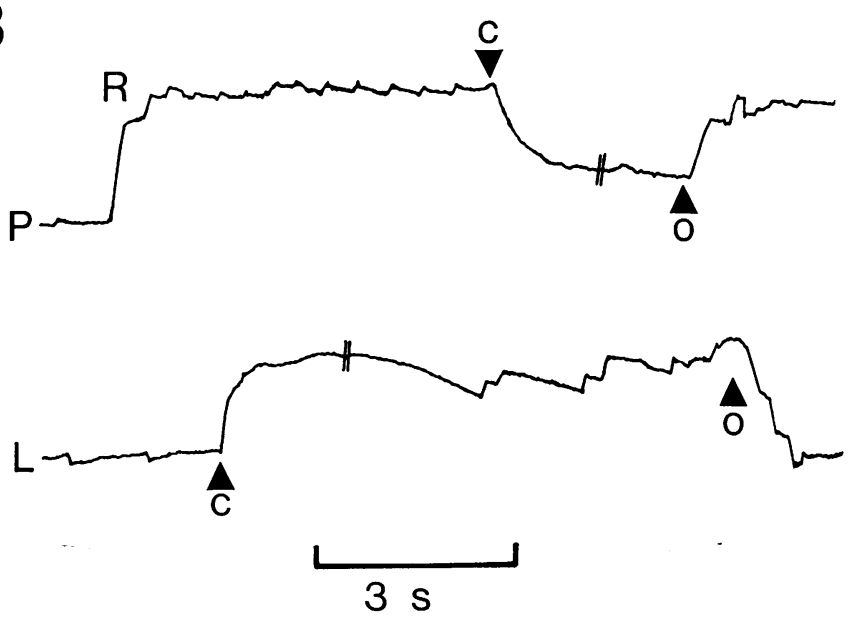

Fig. 3 A. EOG recordings from a patient (case 3 ) with multiple sclerosis. When the eyes fixate a target $30^{\circ}$ to the right a $1^{\circ}$ nystagmus appears. Eye closure produces a rapid drift towards centre and abolishes the nystagmus. With eye closure (C) on primary gaze $(\mathrm{P})$ there is a movement to the right with the appearance of a right-beating nystagmus. Eye closure (C) on left gaze $(\mathrm{L})$ produces a rapid movement to centre with the appearance of a right-beating nystagmus.

B. EOG recordings from a patient (case 4) with a progressive cerebellar syndrome, probably due to multiple sclerosis. $A$ right-beating nystagmus is evident on primary $(\mathrm{P})$ and right (R) gaze; on left (L) gaze there is a left-beating nystagmus. On eye closure $(\mathrm{C})$ there is a rapid movement of the eyes to the centre. On left (L) gaze a right-beating nystagmus develops with eye closure (C). EOG drift negligible. $\mathrm{O}=$ eyes open.

new neutral position. When nystagmus was present during drifting eye movements, either the fast or slow phase could be in the direction of the drift (compare Fig. 2B with Fig. 1B). Of the 9 patients with drifting movements of the eyes all had gaze nystagmus and 8 had additional failure of gaze maintenance. Four patients had bilateral drift, the remainder had unilateral drift.

OTHER EYE MOVEMENT DISORDERS

Of the patients surveyed 14 had centrifugal nystagmus on lateral gaze, 10 had deranged pursuit movements, 7 had exaggerated caloric responses, and 6 had rebound nystagmus.
COMPARISON WITH THE CENTRIPETAL NYSTAGMUS AND EYE MOVEMENT DRIFT RESULTING FROM UNILATERAL LOSS OF LABYRINTHINE FUNCTION

Centripetally beating nystagmus is most commonly encountered in patients with loss of labyrinthine function when gaze is directed towards the side of the lesion. A typical nystagmus resulting from loss of end-organ function is illustrated in Fig. 4. The nystagmus is of a large-amplitude, saw-tooth type. The fast phase of the nystagmus carries the eyes towards the side of the intact labyrinth. In the presence of vision the nystagmus may be partly suppressed. In the absence of visual control of eye 


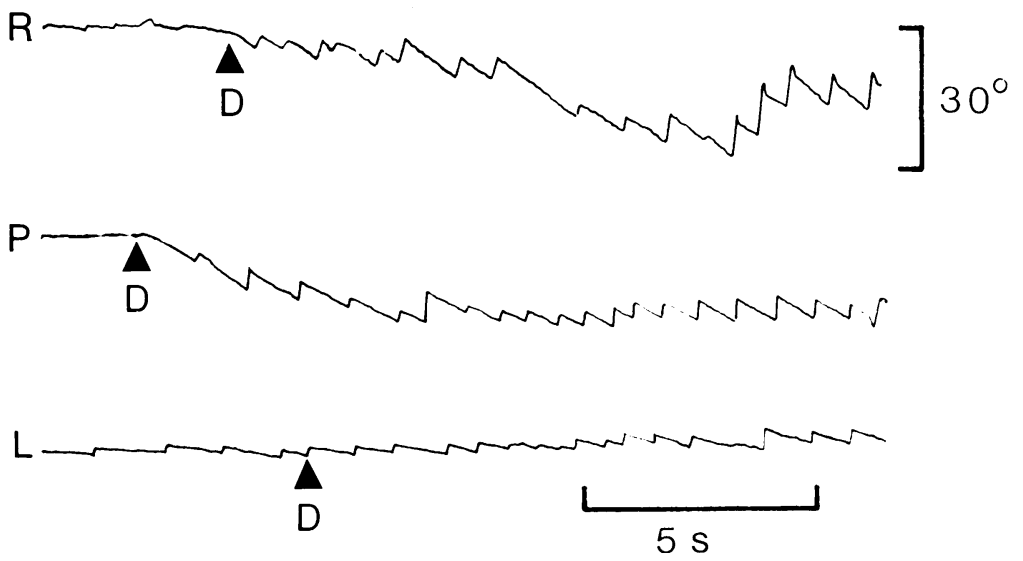

Fig. 4 EOG recordings of eye movements in the horizontal plane in a patient with total loss of function of the left vestibular end organ. $\mathrm{R}, \mathrm{L}$, and $\mathrm{P}$ indicate that eyes are fixated on a target $30^{\circ}$ to the right, left, or in primary gaze, respectively. At $\mathrm{D}$ the patient is in total darkness. EOG drift negligible.

position there is an overall drift of the eyes in the direction of the slow phase. The velocity of the drift, as of the slow phase of the nystagmus, is inversely proportional to the magnitude of the displacement of the eyes away from the side of the intact labyrinth. When the gaze is towards the side of the absent labyrinth, the nystagmus beats centripetally.

Drifting eye movements and centripetal nystagmus in peripheral lesions may be distinguished from the similar eye movement signs which we have described in association with cerebellar disease in the following respects: (1) A peripheral nystagmus at any one time is unidirectional for all positions of gaze. In the absence of optic fixation a centripetal nystagmus in cerebellar disease may replace a centrifugal fixation nystagmus present in the same direction of gaze. (2) Drift in peripheral lesions is unidirectional. In cerebellar disease the drift may consist of alternate wanderings in either direction, although it may be unidirectional. (3) The slow phase of centripetal nystagmus in cerebellar disease may be in the opposite direction to that of the overall eye movement drift. In peripheral lesions drift and slow-phase movements are in the same direction.

In cases of unilateral loss of labyrinthine function partial recovery of end-organ function occurs after some time, and the peripheral nystagmus may reverse so that the fast phases are executed in the direction of the deranged labyrinth (Stenger, 1959). Although this is rare, when it does occur EOG records can be confusing, so alternative methods, such as caloric irrigation, must be used to establish the state of the vestibular end organs before a nystagmographic interpretation can be given.

\section{Discussion}

Westheimer and Blair (1974) have described the abnormalities of eye movement following cerebellectomy in the monkey, and these are particu- larly relevant to an understanding of our clinical findings. Immediately after hemicerebellectomy in mature animals the eyes fail to maintain lateral gaze (ipsilateral to the lesion) and drift centripetally. About a week later a compensatory bias towards the side of the lesion develops to counteract the gaze failure. The bias is present in all positions of gaze and is manifest as a drifting eye movement. If the eyes drift, visual (in the presence of fixation) and brain stem mechanisms which monitor eye movement sense the drift, and they correct for it by generating saccades, the net result being nystagmus. Hence the hemicerebellectomised adult monkey has a bilateral gaze nystagmus, due to gaze paresis ipsilaterally and to compensatory bias contralaterally. Any imbalance in the net sum of pathological and compensatory drift may result in an offsetting of the neutral resting position of the eyes. This shift of the 'rest point' of Holmes (1917), who observed it in lesions of the cerebellum, or the wandering 'neutral point' of Robinson (1974), who found it in cerebellectomised cats, is evident in some of our patients.

\section{GAZE FAILURE}

Fourteen of our patients had gaze nystagmus, of whom 13 had bilateral gaze failure in darkness or on eye closure. It is well known that eye closure produces a wide range of effects in the central nervous system. In our patients eye closure appears to shut down the brain stem mechanisms which normally monitor gaze position and correct it by generating fast phases of nystagmus and compensatory biases. The result is a failure of gaze maintenance, which when total implies additional brain stem lesions.

\section{DRIFTING MOVEMENTS OF THE EYES}

IN DARKNESS OR UPON EYE CLOSURE

There are several possible sources of eye movement drift. In normal subjects a small centripetal drift is 
found with extreme lateral gaze in darkness (Becker and Klein, 1973). It is the presumed role of the cerebellum to generate a centrifugal compensatory bias to counteract this centripetal drift. The interplay between centripetal and centrifugal drift is shown by the fact that rebound nystagmus can be demonstrated in normals after prolonged eccentric fixation (Zee et al., 1976).

In acute cerebellar lesions the balance between both centripetal and centrifugal drift is deranged. The centripetal drift is strong, even during fixation, resulting in ipsilateral fixation nystagmus (Holmes, 1917). It can be assumed that the counterbalancing centrifugal drift is lost immediately after the cerebellar lesion. In subacute and chronic cerebellar lesions, however, a compensating centrifugal drift can reappear. Robinson (1974) has shown that in the cerebellectomised cat the eyes wander in darkness. This has been interpreted as a failure to detect and nullify the low frequency noise of the oculomotor system with a consequent wandering of the neutral position point.

The drifting eye movements observed in patients with cerebellar disease closely resemble those found in animals following experimental lesions of the cerebellum, so it is likely that they can be attributed to the same mechanisms. Drift in a patient with a cerebellar lesion represents the algebraic summation of drift movements due to natural centripetal drift from eccentric gaze, loss of bias produced by the cerebellum to compensate for natural drift, drift caused by the development of new compensatory biases, and wandering of the neutral point due to low-frequency noise.

\section{CENTRIPETAL NYSTAGMUS}

When the eyes drift, a nystagmus is likely to occur, and centripetally beating nystagmus is generated when the direction of drift is away from primary gaze. As indicated above, eye movement drift is the result of summing pathological and compensatory biases. When this sum directs the eyes centrifugally, they drift outwards, producing the centripetal nystagmus. The lack of centrifugal drift in normal subjects, and the fact that it takes time to develop after a cerebellar lesion, suggest that when it occurs it is a sign of compensation. In cases of over-compensation centrifugal drift produces centripetal nystagmus. In matching compensation it induces the decay of gaze paretic nystagmus and the appearance of rebound nystagmus (Hood et al., 1973; Baloh et al., 1975; Zee et al., 1976). The explanations offered for the phenomena of 'rebound' nystagmus (Hood et al., 1973; Zee et al., 1976) and 'periodic alternating nystagmus' (Daroff and Dell'Osso, 1974; Rudge and Leech, 1976) are similar to those involved to account for the drifting eye movements and associated nystagmus observed in the present study. The possibility exists that they are attributable to manifestations of the same basic mechanisms.

\section{ORIGIN OF COMPENSATORY DRIFT}

Visual mechanisms of fixation predominate and have the ability to rapidly 'switch off' centrally generated eye movement. This is clearly shown in Fig. 1B, in which the sudden appearance of a target returns the eyes to the lateral gaze and totally inhibits centripetal nystagmus. The situation is similar in 'recovery' nystagmus, in which rapid head shaking can immediately reverse its direction (Stenger, 1965). This is evidence that the mechanism responsible for producing compensatory 'biases' may be a common one.

Westheimer and Blair (1974) demonstrated the origin of the compensatory drift to be extracerebellar, the primary candidate being the vestibular nuclei. Indeed in many ways the phenomena seen after clinical and experimental cerebellar lesions are similar to the recovery phenomena observed after peripheral vestibular lesions (Stenger, 1959).

Recent neurophysiological evidence (Baker et al., 1976; Baker et al., 1977) would implicate the nucleus prepositus hypoglossi as an alternative candidate.

\section{Conclusions}

Certain eye movement signs have been systematically associated with cerebellar disease. These are (1) failure to maintain lateral gaze, (2) drifting eye movements in darkness or on closure, (3) offsetting of the neutral resting position of the eyes and gaze nystagmus which beats centripetally. Similar disorders of eye movements are scen in adult animals with experimental lesions of the cerebellum. All these disorders can be explained on the simple hypothesis that the cerebellum helps to maintain lateral gaze and that brain stem mechanisms monitor gaze position generating compensatory biases in the absence of normal cerebellar function.

\section{APPENDIX}

CASE 1 (see Fig. 1)

Vascular accident involving cerebellum and probably brain stem on left side

Three months before admission a 68-year-old man suddenly felt unsteady and giddy, and veered to the left on walking. Over the next few days he improved, but a week later he had a similar attack to the first and vomited profusely. He subsequently remained unsteady.

On admission to the National Hospital (Dr M. J. McArdle) the cranial nerves were found to be normal. Tone was normal in the upper limbs but slightly increased 
in the right leg. Power was normal. There was mild impairment of finger/nose/finger and heel/shin/knee testing on the left. Tendon reflexes were rather brisk, more in the right arm than the left, and were symmetrically brisk in the lower limbs. Plantars were flexor. The patient was unsteady and tended to veer to the left on walking. There was no sensory abnormality. Blood pressure was raised at $150 / 110 \mathrm{mmHg}$.

An air encephalogram and cerebrospinal fluid examination were normal. Formal neuro-otological examination revealed fine first-degree nystagmus to both sides, with rebound nystagmus. There was no positional nystagmus, and optokinetic responses were normal. There was a suggestion of a mild left canal paresis.

CASE 2 (see Fig. 2)

\section{Left-sided cerebellar and fourth ventricular tumour}

removed 15 years prior to study

The patient presented at the age of 8 with headache, vomiting, and ataxia. He was admitted under the care of Professor Valentine Logue to Maida Vale Hospital. Examination at that time showed normal optic discs, a left sixth nerve palsy, some facial asymmetry-the right being lower than the left-first-degree nystagmus greater to the left than the right, and marked left-sided ataxia.

Ventriculography revealed symmetrical dilatation of the lateral ventricles, with displacement of the fourth ventricle to the right. At operation a low-grade cystic astrocytoma was found on the left side of the fourth ventricle, extending into the cerebellar hemisphere. Virtual total removal was achieved, apart from a small plaque of tissue on the floor of the fourth ventricle. After a course of radiotherapy all the symptoms resolved apart from a mild left-sided ataxia. Subsequently he developed episodes of unilateral headache associated with nausea, but did not consult his physician for the next 15 years, when his headaches became more troublesome, and he was referred back to the hospital. Examination revealed mild left-sided ataxia but no other convincing signs. EMI scan showed left cerebellar atrophy consistent with removal of the tumour and no evidence of recurrence. This was confirmed on lumbar air encephalography.

On formal neuro-otological testing he had mild leftsided perceptive deafness. Extraocular following movements were broken up, there was first-degree nystagmus to the left, and bilateral rebound nystagmus was present. There was a directional preponderance of the optokinetic nystagmus and caloric responses to the left.

\section{CASE 3 (see Fig. 3)}

Multiple sclerosis with evidence of cerebellar dysfunction At 44, the patient developed paraesthesiae in both feet, which resolved over a period of 3 months. Approximately 6 months later the paraesthesiae recurred and then involved the left hand as well as the feet. At about the same time he noted some imbalance. These symptoms persisted for 1 year and he developed increasing unsteadiness of gait, with weakness of the legs over a period of 2 months. He was admitted to the National Hospital, Queen Square (Professor R. W. Gilliatt).
On examination at that time the cranial nerves were normal. In the upper limbs tone was increased on the left and there was a mild pyramidal weakness of that limb. There was bilateral finger/nose ataxis greater on the left than the right. In the lower limbs he had a spastic paraparesis, greater on the left, and moderate ataxia. His gait was markedly ataxic. Reflexes were brisk, plantar responses flexor, and the abdominal reflexes present. On sensory testing there was subjective superficial sensory impairment of the left hand and of the feet, with impairment of discrimination between two points in the left index finger.

Visual evoked potentials to a pattern stimulus were bilaterally delayed, indicating the presence of old optic neuritis. Formal neuro-otological examination showed directional preponderance of the optokinetic nystagmus to the left.

\section{CASE 4 (see Fig. 3)}

Progressive cerebellar ataxia, probably due to multiple sclerosis

A 30-year-old woman presented to the National Hospital, Queen Square (Dr Macdonald Critchley), with a $3 \frac{1}{2}$-year history of progressive deterioration of gait, difficulty in using the right hand, and slurred speech. There was no family history of neurological disorder.

Examination at that time showed marked cerebellar dysarthria. In the limbs tone was normal, but there was a mild weakness of finger and wrist extension, greater on the right, together with some weakness of hip flexion on the right. Co-ordination was moderately impaired in the upper limbs and grossly so in the lower. Reflexes were normal in the upper limbs and depressed or absent in the lower. Plantar responses were equivocal and the abdominal reflexes present. The gait was grossly ataxic, such that the patient was unable to stand without support. Sensation was intact.

Formal neuro-otological testing showed seconddegree nystagmus to the right, first-degree to the left, and first-degree upwards. Following movements were broken up in the horizontal plane. There was a central type of positional nystagmus. Caloric responses were greatly enhanced but symmetrical. The optokinetic responses were grossly deranged in all directions, with almost complete suppression. Cerebrospinal fluid had a strongly paretic Lange curve, with negative serological testing for syphilis.

In spite of the progressive history it was concluded that the patient had multiple sclerosis in view of the spinal fluid findings.

\section{References}

Baker, R., Gresty, M., and Berthoz, A. (1976). Neuronal activity in the prepositus hypoglossi nucleus correlated with vertical and horizontal eye movement in the cat. Brain Research, 101, 366-371.

Baker, R., Berthoz, A., and Delgado-Garcia, J. (1977). Monosynaptic excitation of trochlear motor neurons following electrical stimulation of the prepositus hypoglossi nucleas. Brain Research, 121, 157-161.

Baloh, W., Konrad, H. R., and Honrubia, V. (1975). Vestibulo-ocular function in patients with cerebellar atrophy. Neurology, 25, 160-168. 
Becker, W., and Klein, H. H. (1973). Accuracy of saccadic eye movements and maintenance of eccentric eye positions in the dark. Vision Research, 13, 1021-1034.

Daroff, R. B., and Dell'Osso, L. F. (1974). Periodic alternating nystagmus and the shifting null. Canadian Journal of Otolaryngology, 3, 367-371.

Holmes, G. (1917). Disturbances of the ocular movements and nystagmus. Brain, 40, 500-505.

Hood, J. D., Kayan, A., and Leech, J. (1973). Rebound nystagmus. Brain, 96, 483-502.

McAlpine, D., Lumsden, C. E., and Acheson, E. D. (1972). Multiple sclerosis, a Reappraisal. Livingstone: Edinburgh.

Orzechowski, C. (1927). De l'ataxie dysmetrique des yeux. Neurologie, 35, 1-18.

Robinson, D. A. (1974). The effect of cerebellectomy on the cat's vestibulo-ocular integrator. Brain Research, 71, 195-207.
Rudge, P., and Leech, J. (1976). Analysis of a case of periodic alternating nystagmus. Journal of Neurology, Neurosurgery, and Psychiatry, 34, 314-319.

Stenger, H. H. (1959). 'Erholungnystagmus' nach einseitigem Vestibularisausfail, ein dem Bechterew-Nystagmus verwandter Vorgang. Archiv für Ohren-, Nasen- und Kehlkopfheilkunde, 175, 545-549.

Stenger, H. H. (1965). Schwindelanalyse, Untersuchung auf Spontan- und Provokations-nystagmus HNO Heilkunde. Ed. Berendes, Link and Zollner, Vol. III, Part 1, pp. 540580.

Westheimer, G., and Blair, S. M. (1974). Functional organization of primate oculomotor system revealed by cerebellectomy. Experimental Brain Research, 21, 463-472.

Zee, D. S., Yee, R. D., Cogan, D. G., Robinson, D. A., and Engel, W. K. (1976). Ocular motor abnormalities in hereditary cerebellar ataxia. Brain, 99, 207-234. 